\title{
Two distinct halo populations in the solar neighborhood: evidence from stellar abundance of beryllium
}

\author{
Kefeng Tan and Gang Zhao \\ Key Laboratory of Optical Astronomy, National Astronomical Observatories, Chinese \\ Academy of Sciences, Beijing 100012, China \\ email: tan@nao.cas.cn; gzhao@nao.cas.cn
}

\begin{abstract}
It is now generally believed that the Galaxy was formed through hierarchical merging, which means that different components of the Galaxy may have experienced different chemical evolution histories. Since alpha elements are mainly produced by core collapse supernovae, they are closely associated with the star formation history of the Galaxy. In this regard, Galactic components with different alpha elemental abundance patterns may show different behaviors in beryllium abundances since the production of beryllium is correlated with the cosmic rays and thus the supernovae. A recent study by Nissen \& Schuster (2010) has revealed the existence of two distinct halo populations in the solar neighborhood based on the alpha elemental abundances and kinematics of 94 dwarf stars. We determined beryllium abundances for some of these stars and find systematic differences in beryllium abundances between these two halo populations. Our results consolidate the conclusion of two distinct halo populations in the solar neighborhood. Our results also show that beryllium abundance is a very good indicator of star formation rate, and could be used to trace the substructures of the Galactic halo.
\end{abstract}

Keywords. Galaxy: formation, Galaxy: halo, stars: abundances

\section{Introduction}

The fourth element in the periodic table, beryllium (Be), has very special origins. The Standard Big Bang Nucleosynthesis could produce very little Be. Furthermore, Be cannot be produced by nucleosynthesis in stellar interiors, because the threshold temperature for nuclear fusion of Be is relatively very low. It is now widely accepted that most of the Be observed today is produced by the spallation reactions between the cosmic-rays (CRs) and interstellar medium (ISM). The general picture is that, during the collisions between the CRs and ISM, carbon, nitrogen, and oxygen nuclei split into lighter elements, such as lithium, beryllium, and boron (Reeves et al. 1970; Duncan et al. 1997). Therefore, the production rate of $\mathrm{Be}$ is dependent on the flux of CRs. We know that the Galaxy was formed through hierarchical merging, and different components of the Galaxy may have experienced different chemical evolution histories. In this regard, star formation rates, supernovae rates, and thus CRs fluxes for different components of the Galaxy are not necessarily the same. Therefore, we may expect that Be abundance patterns of different Galactic components may not be the same.

\section{Sample and results}

The work by Nissen \& Schuster (2010) (hereafter NS10) provide us with a very good sample to test this prediction. They performed a precise abundance analysis for a sample 

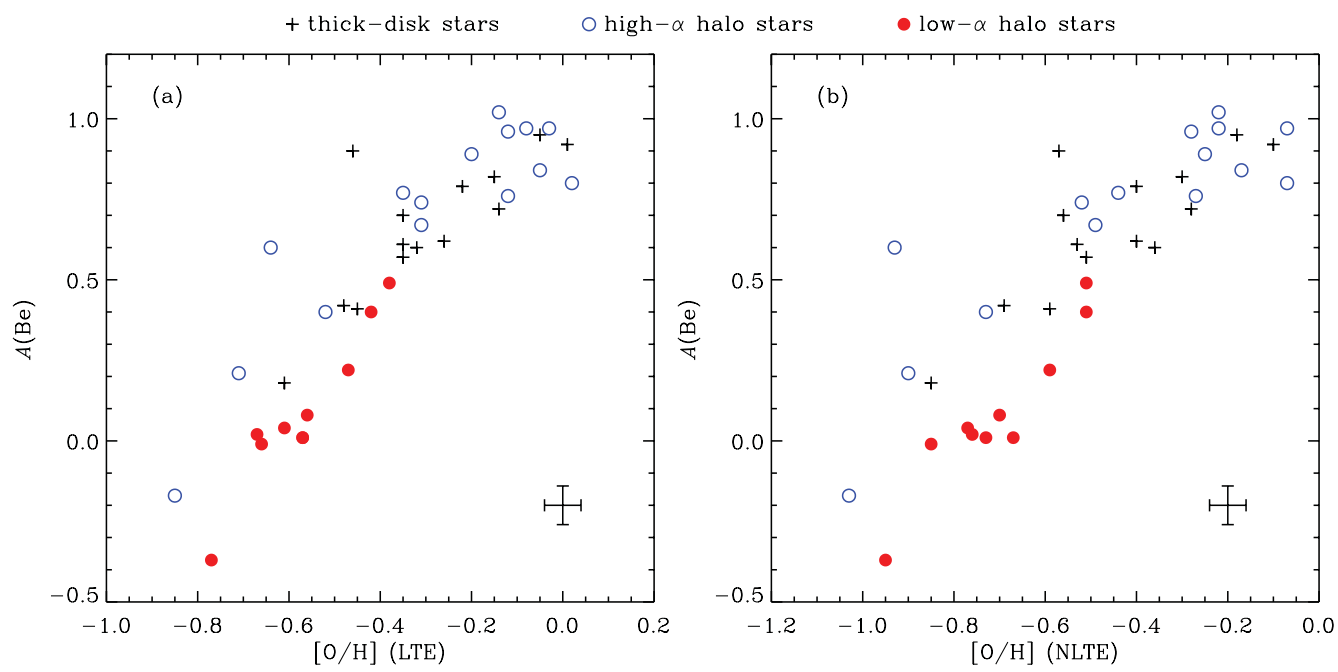

Figure 1. Be abundance as a function of $[\mathrm{O} / \mathrm{H}]$ based on (a) LTE and (b) NLTE analysis.

of 94 thick-disk and halo stars in the solar neighborhood, and found that the halo stars clearly separate into two distinct populations, i.e., the low- and high- $\alpha$ halo populations. The very high precision of stellar parameters make the sample of NS10 ideal for studying the possible different Be abundance patterns for different Galactic components in a systematic way. Therefore, we determined Be abundances for the stars with high-resolution and high signal-to-noise ratio spectra covering the Be II $3130 \AA$ regions in the sample of NS10. Oxygen abundances for these stars are adopted from the results of Ramírez et al. (2012). Both the Be and $\mathrm{O}$ abundances are derived based on the stellar parameters from NS10.

Figure 1 shows $\mathrm{Be}$ abundance as a function of $[\mathrm{O} / \mathrm{H}]$ for the sample stars. It can be seen that $\mathrm{Be}$ abundances of the high- $\alpha$ halo stars are systematically higher than that of the low- $\alpha$ halo stars. As suggested by NS10, the high- $\alpha$ halo stars may form in regions with rapid chemical evolution, while the low- $\alpha$ halo stars may originate from dwarf galaxies with lower star formation rates. In this case, the energy spectrum for the CRs may be different in the regions where the low- and high- $\alpha$ halo stars formed, and thus the production rate of Be could be different (as the effective spallation crosssections are dependent on the energies of the CRs). Therefore, systematic differences in Be abundances between the low- and high- $\alpha$ halo stars can be a natural prediction from the explanation of NS10. In summary, our results consolidate the conclusion of two distinct halo populations in the solar neighborhood by NS10. Moreover, our results show that Be abundance is a very good indicator of star formation rate, and could be used to trace the substructures of the Galactic halo.

\section{Acknowledgments}

This work is supported by the National Natural Science Foundation of China under grant Nos. 11103034 and 11233004. 


\section{References}

Duncan, D. K., Primas, F., Rebull, L. M., Boesgaard, A. M., Deliyannis, C. P., Hobbs, L. M., King, J. R., \& Ryan, S. G. 1997, ApJ, 488, 338

Nissen, P. E., \& Schuster, W. J. 2010, A\&SA, 511, L10

Ramírez, I., Meléndez, J., \& Chanamé, J. 2012, ApJ, 757, 164

Reeves, H., Fowler, W. A., \& Hoyle, F. 1970, Nature, 226, 727 